\title{
Study of the transport properties of annealed (Ga,Mn)As by x-ray absorption spectroscopy
}

\author{
C. J. Ji, ${ }^{\text {a) }}$ X. C. Cao, Q. F. Han, K. Qiu, F. Zhong, and X. H. Li \\ Key Laboratory of Material Physics, Institute of Solid State Physics, Chinese Academy of Sciences, Hefei, \\ Anhui 230031, China \\ H. T. He and J. N. Wang \\ Department of Physics, Hong Kong University of Science and Technology, Clear Water Bay, Kowloon, \\ Hong Kong, China \\ Y. Q. Wang \\ Key Laboratory of Material Physics, Institute of Solid State Physics, Chinese Academy of Sciences, Hefei, \\ Anhui 230031, China
}

(Received 15 March 2007; accepted 11 May 2007; published online 4 June 2007)

\begin{abstract}
A systematic $\mathrm{Mn} L$-edge x-ray absorption is carried out on carefully prepared $\mathrm{Ga}_{0.946} \mathrm{Mn}_{0.054} \mathrm{As}$ ferromagnetic semiconductors with varying $\mathrm{As}_{2} / \mathrm{Ga}$ flux ratio. It is found that the $L_{3}$ peak of the absorption spectroscopy is enhanced after low temperature (LT) annealing. Furthermore it is shown that a more localized electronic structure nearly like the $d^{5}$ high-spin state is obtained. It can be attributed to breaking the $\mathrm{Mn}_{S}-\mathrm{Mn}_{I}$ pairs during the annealing process. Furthermore the authors present a direct evidence for a slightly increase of the Mn substitutional concentration due to LT annealing. (C) 2007 American Institute of Physics. [DOI: 10.1063/1.2746070]
\end{abstract}

Recently much attention has been attracted on the III-V diluted magnetic semiconductors due to the possibility of "spintronic" device applications, ${ }^{1}$ in which the spin degree of freedom of the carriers is exploited. ${ }^{2}$ Many efforts have been focused on the ferromagnetic $\mathrm{Ga}_{1-x} \mathrm{Mn}_{x} \mathrm{As}$ system as a typical class of semiconductors with magnetic and optical properties grown by low temperature (LT) molecule beam epitaxy (MBE).$^{3-7}$ In the Mn-doped (Ga,Mn)As, most of the Mn atoms substitute the $\mathrm{Ga}$ sites $\left(\mathrm{Mn}_{S}\right)$. These $\mathrm{Mn}_{S}$ behave as single acceptors and contribute local moments and holes because of the half-filled $3 d$ shell. However, besides $\mathrm{Mn}_{S}$, there are also some $\mathrm{Mn}$ interstitials $\left(\mathrm{Mn}_{I}\right)$ present in the lattice. They act as double donors and may create antiferromagnetically coupled $\mathrm{Mn}_{S}-\mathrm{Mn}_{I}$ pairs, which strongly suppress the established ferromagnetism. Curie temperature $T_{C}$ for asgrown $(\mathrm{Ga}, \mathrm{Mn}) \mathrm{As}$ is in the range of $30-80 \mathrm{~K}$. Several factors which limit $T_{C}$ have been indicated, e.g., the antisite As, interstitial Mn (Refs. 8 and 9) as deep donors compensating the holes in GaMnAs films. It has been pointed out that $T_{C}$ can be increased significantly by postgrowth low temperature annealing. ${ }^{10}$ By annealing with optimal time and temperatures, the $T_{C}$ can be remarkably improved from below 100 to around $150 \mathrm{~K}$. In order to achieve room-temperature ferromagnetic semiconductors, many efforts have been made to investigate the origin of ferromagnetism in GaMnAs. Therefore it is of great importance to study the states of magnetic impurities in these ferromagnetic semiconductors. Usually, an increase of $T_{C}$ via the low temperature annealing is attributed to the reduction of the $\mathrm{Mn}_{I}$ concentration. Although it is not clearly shown what happens to them, there are some suggestions that these removed $\mathrm{Mn}_{I}$ segregate at the GaMnAs surface. In spite of these achievements, a better understanding of the material properties of this system is desired.

${ }^{a)}$ Electronic mail: jcjcs@theory.issp.ac.cn
Due to the localized nature of the $3 d$ valence states, the electronic structure of the $\mathrm{Mn}$ atom in the GaAs host is greatly affected by its neighboring atoms. The x-ray absorption spectroscopy (XAS) is a powerful technique for the direct probing of the $3 d$ valence electronic structure. ${ }^{11,12}$ So we measure XAS of ferromagnetic GaMnAs with different $\mathrm{As}_{2} / \mathrm{Ga}$ flux ratios at the $\mathrm{Mn} L$ edge. The absorption corresponds to a transition from the Mn- $2 p$ core level states to the unoccupied $3 d$ states.

GaMnAs samples studied in this experiment were grown at $250{ }^{\circ} \mathrm{C}$ in a Riber $32 \mathrm{MBE}$ system on (001) semiinsulating $\mathrm{GaAs}$ substrate. However, each of the samples was grown at different $\mathrm{As}_{2} / \mathrm{Ga}$ flux ratios. The range is limited between 2 and 9. The layers were typically $50 \mathrm{~nm}$ thick and the Mn content was chosen to be $5.4 \%$ measured by Philips $\mathrm{X}$-pert high-resolution diffractometer with collimating mirror. The XAS measurements were performed at the National Synchrotron Radiation Laboratory in Hefei, China. The experiments were conducted in ultrahigh vacuum of $10^{-11}$ Torr at room temperature. XAS was measured in total electron yield mode. Synchrotron radiation was monochromatized using a varied-line-spacing plane grating. To remove oxidized surface layers, samples have been washed in concentrated $\mathrm{HCl}$ for a few seconds, then rinsed in de-ionized water, and inserted in UHV system. All measurements were performed at room temperature.

Figure 1 shows XAS spectra across Mn $2 p$ edge for samples (a) 1, (b) 2, and (c) 3 in total electron yield. Samples 1, 2, and 3 were grown at $\mathrm{As}_{2} / \mathrm{Ga}$ flux ratios of 9, 6, and 5 . The spectra are normalized for incident photon flux. The features around 638 and $651 \mathrm{eV}$ correspond to the $L_{3}\left(2 p_{3 / 2}\right.$ $\rightarrow 3 d)$ and $L_{2}\left(2 p_{1 / 2} \rightarrow 3 d\right)$ levels. To compare the data among all samples, we removed a step function background in each curve, as is normally done for $3 d$ transition elements. The as-grown spectra show a nearly free of structures in between the edges and display two broad shoulders around 640.2 and $642 \mathrm{eV}$. The overall line shape does not appear to change 


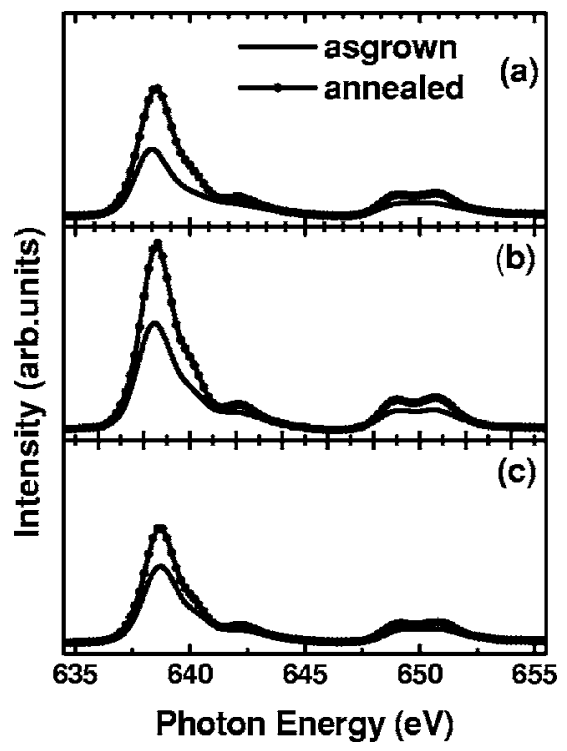

FIG. 1. X-ray absorption spectra in total electron yield detection at the Mn $2 \mathrm{p}$ edge for samples (a) 1 , (b) 2, and (c) 3 . All the samples are annealed at $260{ }^{\circ} \mathrm{C}$ under nitrogen atmosphere for $1 \mathrm{~h}$.

significantly after annealing except for an energy shift of $0.2 \mathrm{eV}$ at $L_{3}$ edge. The most interesting effect is the increase of annealing-induced changes in the intensity of the $L_{3,2}$ spectra. The intensity of the $L_{3}$ peak of sample 2 reaches the maximum. It has attributed the $L_{3}$ peak to the $\mathrm{Mn}_{S}$ in Ref. 13 after removal of the Mn-rich oxide layers. So the increase in the intensity of the XAS is associated with the enhancement of $\mathrm{Mn}_{S}$ component.

Meanwhile the spectra seem similar to the calculated spectrum for the hybridized state reported in Ref. 14, with rather indistinct multiplet splitting compare to $\mathrm{MnO}$, but with a large branching ratio compared to metallic $\mathrm{Mn} .{ }^{15}$ In order to investigate the electronic structure of the GaMnAs film, we estimate the branch ratio for the Mn $L_{3,2}$-edge XAS spectra. The branch ratio $R_{B}$ defined as $R_{B}=\left(A_{2 p 3 / 2}\right) /\left(A_{2 p 3 / 2}\right.$ $\left.+A_{2 p 1 / 2}\right)$, where $A$ is the integrated area under the peaks. $R_{B}$ is strongly influenced by electrostatic interaction and is very sensitive to the change in the electronic structure. As previous study, it reaches the maximum value of $\sim 0.8$ for the $d^{5}$ high-spin state, ${ }^{16}$ while 0.73 for bulk $\operatorname{Mn}\left(d^{5}+d^{6}+d^{7}\right) .{ }^{17}$ As shown in Fig. 2 , all values of the estimated $R_{B}$ are very close to 0.8 after annealing. So we can conclude that the strength of interaction between $\mathrm{Mn}$ atoms changes after annealing. In addition, it has been dictated that vapor Mn shows pure $d^{5}$ high-spin state characteristics for no $\mathrm{Mn}-\mathrm{Mn}$ interaction. ${ }^{18}$ Therefore, it is suggested that the electronic configuration of the GaMnAs film after annealing is more likely to the $d^{5}$ high-spin state. As in our previous study, it has been shown that the antiferromagnetic $\mathrm{Mn}_{S}-\mathrm{Mn}_{I}$ pairs exist in the layers and are broken by annealing. ${ }^{9}$ So in the case of GaMnAs layers, the weakness of interaction can also be interpreted as an effect of removing such pairs. In addition, the Mn $3 d$ orbital is more localized for nearly $d^{5}$ high-spin state.

In Zener mean-field model description, Curie temperature $T_{C}$ is determined by the concentration of holes $p$ and roughly proportional to $p^{1 / 3}$. Therefore higher $T_{C}$ will be reached due to more holes. For this purpose, resistivity $[\rho(T)]$ is measured using standard four-probe technique. Three samples were prepared under the same growth parameters as samples discussed above. They were annealed at Downloaded 06 May 2009 to 143.89.106.125. Redistribution subject

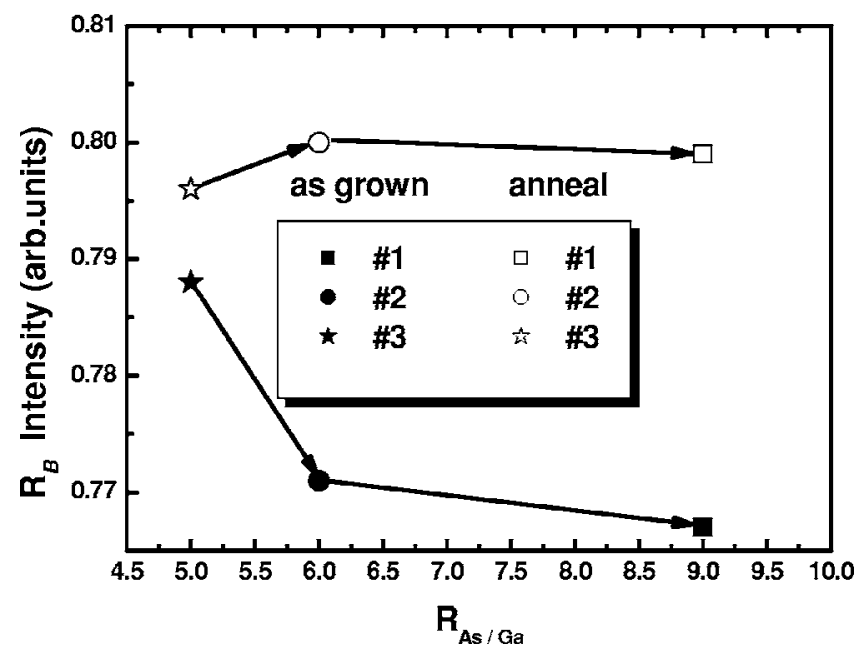

FIG. 2. Branching ratio for as-grown samples is shown as full symbols. Open symbols show branching ratio as annealed samples.

$260{ }^{\circ} \mathrm{C}$ for $1 \mathrm{~h}$ under nitrogen atmosphere. Figure 3 shows the temperature dependence of the resistivity $\rho(T)$, for samples (a) 1, (b) 2, and (c) 3 with $T_{C}$ indicated. Compared with the as-grown sample, the annealed samples show lower resistivity and higher $T_{C}$. It is clearly deemed that the properties of epilayers are superior after annealing. This is expected and consistent with the previous result. ${ }^{10}$ Obvious enhancements take place on sample 2, which also confirms the conclusion of Fig. 1. It is observed that the shape and intensity of $L_{3}$ peak are sharper and stronger than the other two. That means more localized electronic structure and higher concentration of $\mathrm{Mn}_{S}$ composition being obtained in such samples. As antisite are one of major defects in as-grown samples under As-rich growth conditions. These defects play an important role in the low temperature transport properties of GaMnAs films. As is well known, As antisite density depends on the MBE growth conditions and can be adjusted by varying $\mathrm{As}_{2} / \mathrm{Ga}$ flux ratio. Increasing the ratio will also increase the As antisites. Recently, it has been pointed out that there is a balance between $\mathrm{Mn}_{I}$ and As antisites defect, ${ }^{18}$ which affords a way to suppress one type of defects upon

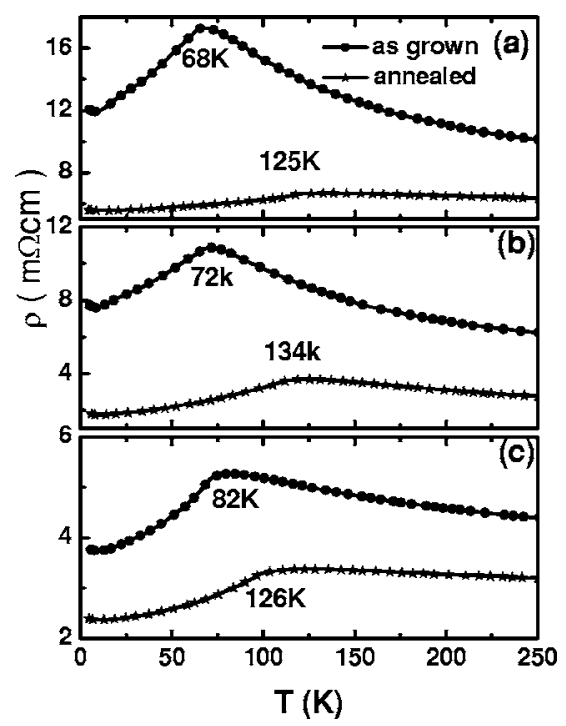

FIG. 3. Temperature dependence of the resistivity $\rho(T)$, for samples (a) 1 , (b) 2, and (c) 3 with $T_{C}$ indicated.

to AIP license or copyright; see http://apl.aip.org/apl/copyright.jsp 


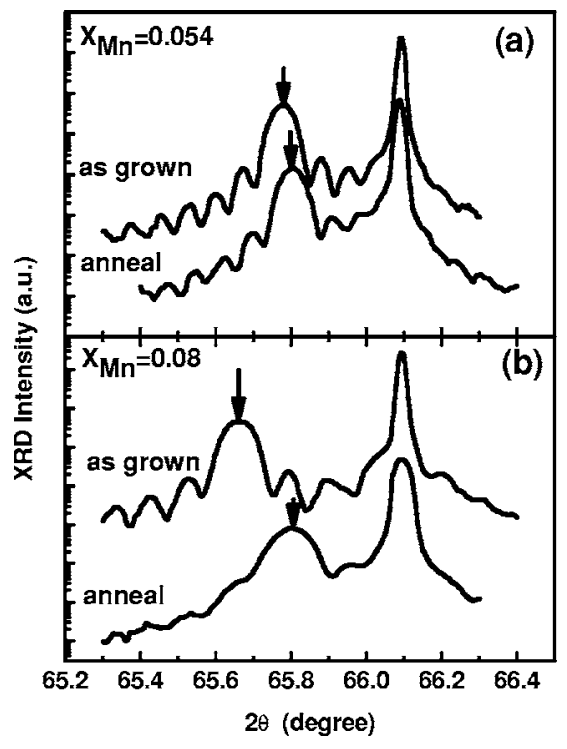

FIG. 4. Difference of HRXRD spectra of $\mathrm{Ga}_{1-x} \mathrm{Mn}_{x}$ As samples with (a) $x$ $=0.054$ and (b) $x=0.08$.

increasing the density of the other defects in as-grown films. ${ }^{19}$ Hence it is deduced that high or low $\mathrm{As}_{2} / \mathrm{Ga}$ flux ratio both weakens the effect of the annealing.

In order to verify the effect of $\mathrm{Mn}_{I}$ on the lattice of GaMnAs, we have used low temperature thermal annealing which is recognized to remove $\mathrm{Mn}_{I}$ defects (but dose not affect $\mathrm{As}_{\mathrm{Ga}}$ antisites). ${ }^{20}$ The resulting lattice parameter change by LT annealing is thus believed to come from the $\mathrm{Mn}_{I}$.

In Fig. 4, we show the difference high-resolution x-ray diffraction (HRXRD) spectra for $\mathrm{Ga}_{1-x} \mathrm{Mn}_{x} \mathrm{As}$ samples with (a) $x=0.054$ and (b) $x=0.08$. For the GaMnAs samples with $5.4 \% \mathrm{Mn}$, the diffraction of peak of the annealed sample shifted a little bit to larger angle $\left(0.050^{\circ}\right)$, which corresponds to the reduced lattice constant of about $0.4 \%$. The total film thickness has no big change if we calculate it from the interference fringes in the XRD spectrum. Judging from the out of the phase interference pattern in the XRD, the film thickness change is near $0.1 \mathrm{~nm}$ (half of the X-ray wavelength). For the sample with $8 \% \mathrm{Mn}$, we find that the peak shift is very large. This is consistent with the fact that there are more $\mathrm{Mn}_{I}$ in this sample due to lower growth temperature employed to incorporate higher Mn contents. ${ }^{21}$

In conclusion, we have investigated the Mn $L$-edge x-ray absorption spectroscopy and resistivity measurements on the as-grown and annealed samples with different $\mathrm{As}_{2} / \mathrm{Ga}$ flux ratios. By discarding the surface contaminants, ${ }^{22}$ it is found that low temperature transport properties can be improved by breaking the $\mathrm{Mn}_{S}-\mathrm{Mn}_{I}$ pairs. Besides, a slightly increase of the substitution $\mathrm{Mn}\left(\mathrm{Mn}_{S}\right)$ components is observed. The pursuit of increasing the Curie temperature, where postgrowth annealing has shown to be very important, has led to an increasingly deeper understanding of $(\mathrm{Ga}, \mathrm{Mn}) \mathrm{As}$.

This work was supported by the Knowledge Innovation Program of Chinese Academy of Sciences. Authors J.N.W. and H.T.H. would like to acknowledge the financial support of the Research Grant Council of Hong Kong SAR via Grant Nos. 603704 and 602906.

${ }^{1}$ S. A. Wolf, D. D. Awschalom, R. A. Buhrman, J. M. Daughton, S. von Molnár, M. L. Roukes, A. Y. Chtchelkanova, and D. M. Treger, Science 294, 1488 (2001).

${ }^{2}$ Y. Ohno, D. K. Young, B. Beschotsen, F. Matsukura, H. Ohno, and D. D. Awschalom, Nature (London) 402, 790 (1999).

${ }^{3}$ H. Ohno, A. Shen, F. Matsukura, A. Oiwa, A. Endo, S. Katsumoto, and Y. Iye, Appl. Phys. Lett. 69, 363 (1996).

${ }^{4}$ T. Hayashi, M. Tanaka, T. Nishinaga, H. Shimada, H. Tsuchiya, and Y. Otuka, J. Cryst. Growth 175, 1063 (1997).

${ }^{5}$ A. Van Esch, L. Van Bockstal, J. De Boeck, G. Verbanck, A. S. van Steenbergen, P. J. Wellman, B. Grietens, R. Bogaerts, F. Herlach, and G. Borghs, Phys. Rev. B 56, 13103 (1997).

${ }^{6} \mathrm{H}$. Ohno, Science 281, 951 (1998).

${ }^{7}$ T. Dietl, H. Ohno, F. Matsukura, J. Cibert, and D. Ferrand, Science 287, 1019 (2000).

${ }^{8}$ P. A. Korzhavyi, I. A. Abrikosov, E. A. Smirnova, L. Bergqvist, P. Mohn, R. Mathieu, P. Svedlindh, J. Sadowsk, E. I. Isaev, Yu. Kh. Vekilov, and O. Eriksson, Phys. Rev. Lett. 88, 187202 (2002).

${ }^{9}$ H. T. He, C. L. Yang, W. K. Ge, J. N. Wang, X. Dai, and Y. Q. Wang, Appl. Phys. Lett. 87, 162506 (2005).

${ }^{10}$ T. Hayashi, Y. Hashimoto, S. Katsumoto, and Y. Iye, Appl. Phys. Lett. 78, 1691 (2001).

${ }^{11}$ G. van der Laan and I. W. Kirkman, J. Phys.: Condens. Matter 4, 4189 (1992).

${ }^{12}$ F. M. F. de Groot, J. C. Fuggle, B. T. Thole, and G. A. Sawatzky, Phys. Rev. B 42, 5459 (1990).

${ }^{13}$ K. M. Yu, W. Walukiewicz, T. Wojtowicz, J. Denlinger, M. A. Scarpulla, X. Liu, and J. K. Furdyna, Appl. Phys. Lett. 86, 042102 (2005).

${ }^{14}$ K. W. Edmonds, N. R. S. Farley, R. P. Campion, C. T. Foxon, B. L. Gallagher, T. K. Johal, G. van der Laan, M. MacKenzie, and J. N. Chapman, Appl. Phys. Lett. 84, 4065 (2004).

${ }^{15}$ Y. Yonamoto, T. Yokoyama, K. Amemiya, D. Matsumura, and T. Ohta, Phys. Rev. B 63, 214406 (2001).

${ }^{16}$ B. T. Thole and G. van der Laan, Phys. Rev. B 38, 3158 (1988).

${ }^{17}$ R. D. Cowan, The Theory of Atomic Structure and Spectra (University of California, Berkeley, 1981), Chap. 20, pp. 613-615.

${ }^{18}$ J. Sadowski and J. Z. Domagala, Phys. Rev. B 69, 075206 (2004).

${ }^{19}$ P. Mahadewan and A. Zunger, Phys. Rev. B 68, 075205 (2003).

${ }^{20}$ K. W. Yu, W. Walukiewiez, T. Wojtowicz, I. Kuryliszyn-Kudelska, X. Liu, Y. Sasaki, and J. K. Furdyna, Phys. Rev. B 65, 201303 (2002).

${ }^{21}$ R. P. Campion, K. W. Edmonds, L. X. Zhao, K. Y. Wang, C. T. Foxon, B. L. Gallagher, and C. R. Staddon, J. Cryst. Growth 247, 42 (2003).

${ }^{22}$ D. Wu, D. J. Keaveny, Ruqian Wu, E. Johnston-Halperin, D. D. Awschalom, and Jing Shi, Phys. Rev. B 71, 153310 (2005). 